\title{
Five Reasons To Care About Mobile Cloud Computing
}

\author{
Fabrizio Capobianco ${ }^{a}$ \\ (a) CEO, Funambol Inc, Free Software entrepeneur
}

DOI: $10.5033 /$ ifosslr.v1i2.24

\begin{abstract}
Funambol Inc.'s Fabrizio Capobianco explores the technical and market opportunities presented by the intersection of cloud technologies and rapid advances in mobile computing for Free \& Open Source software. In doing so he exposes the unique needs of this new market segment, and the legal challenges which may arise from its development.
\end{abstract}

\section{Keywords}

Cloud computing, mobile computing, AGPL

\section{Info}

This item is part of the Tech Watch section of IFOSS L. Rev. For more information, please consult the relevant section policies statement.

Mobile cloud computing represents an opportunity for the free/libre open source software movement that is just as big and radical as cloud computing, maybe even more so.

The term 'cloud computing' has been defined by the U.S. Government's National Institute of Standards and Technology ${ }^{1}$ as "a model for enabling ubiquitous, convenient, on-demand network access to a shared pool of configurable computing resources (e.g., networks, servers, storage, applications, and services) that can be rapidly provisioned and released with minimal management effort or service provider interaction."

There are ongoing efforts to standardize cloud computing (Open Cloud Manifesto) but they seem to lack use cases about mobile computing. Cloud computing becomes mobile when a mobile device tries to access a shared pool of computing resources on-demand. There are at least 5 reasons why mobile cloud computing is important for free and open source software.

\section{Mobile cloud computing is big in size}

At the end of 2009, mobile phones were four billion. By 2013, that number is projected to grow to 6 billion. That is many times the number of personal computers. And when we start including in the mobile world other Internet capable devices, like ebook readers, photo frames, printers, photo and video cameras, personal navigators, the numbers go way up. Small portable devices that can

1 Full document of the NIST Cloud Computing http://csrc.nist.gov/groups/SNS/cloud-computing/. 
access information are already part of everyday life for hundreds of millions of people in the developed world. Also, many hints point to the fact that developing countries will be using the mobile cloud before they get to the 'regular' one.

Just as Free/Libre Open Source Software played a major role in the growth of the Internet and cloud computing, sparking issues about openness and freedom, the Free Software movement has the potential to provide a similar yet different impact on mobile cloud computing.

\section{Mobile cloud computing is a need - form factor and other needs}

By definition, mobile devices that access the Internet are performing mobile cloud computing: handsets need to borrow storage and computing power from the cloud because of their limited resources or because it makes more sense. For example, consider modern wireless car navigators, like the Dash: these devices not only can store locally the maps and calculate routes, but they rely on the cloud to get real time information about traffic conditions and plan the routes accordingly. Accessing data in the cloud from mobile devices is becoming a basic need.

\section{Mobile cloud needs interoperability}

Mobile cloud services are largely dominated by vendor specific walled gardens, and debate is not as intense as one would expect given the numbers of cell phone users. Probably this is due to the fact that not only Free Software powered mobile phones are still a minority, but also installing new software on phones was not an option for the mass market until recently. After iPhone and Android, with more and more 'application stores' emerging, the issue of mobile users' freedom is showing up. Users of one handset, for example, may want to get their email from a provider but sync pictures with another. Or if they buy music from a digital store from the desktop computer, they want to sync their playlists with any phone.

A minimum requirement is interoperable services ${ }^{2}$ implementing open standards, because users' data must be preserved at all costs. Proprietary walled gardens create small monopolies that sometimes grow big and take away personal data from the users. Consider these recent cases that demonstrate that users of mobile cloud services are exposed to serious problems.

Palm Pre owners cannot access the music that they bought and stored in Apple's iTunes: Apple still wants to own the music it sold its users and keep their data hostage. Similar risks are run by owners of Amazon Kindle, who had their purchased books deleted too easily by Amazon from the devices.

The recent fiasco with Microsoft losing people's data is the opening act of how we've all learned that data is not necessarily safe in a proprietary cloud. If one of the world's premier software companies cannot be trusted to keep people's data safe, who can be trusted? Furthermore, do people really want Microsoft, Google et al to access all their data? With the cloud in general and in particular, the mobile cloud (because you want your mobile data backed up), it is more important than ever that people have the full ability to access and preserve their data, which means the open mobile cloud. These are just visible signals of proprietary services battling to own user data. If iTunes and Microsoft used interoperable and open standards, which could be safely implemented in free/libre open source software, their users would not face these problems.

2 http://maffulli.net/2009/05/19/locked-devices-gplv3-and-the-path-to-mobile-freedom/ 


\title{
4. Mobile cloud largely depends on locked-down devices
}

Network operators don't want users to be too free, so most of them prevent users to run applications that are not digitally signed. RIM, Apple and to some extent, Symbian devices are locked down, which renders users' freedom in the mobile cloud a balancing act: on one hand a developer needs to obey the rules dictated by network operators and device manufacturers; on the other hand the same developer needs to find ways to deliver freedom to users. In Funambol's case, for example, the official iPhone client can only sync contacts because the official Apple SDK only allows that. Nonetheless, the Funambol client for iPhone can also sync calendars by accessing directly the sqlite database, but this version cannot run on the device unless it is unlocked (breaking Apple's warranty).

\section{Mobile cloud is an opportunity for free software providers}

With so many new mobile devices hitting the market, billions of new users have the issue of freedom for the software on the device and freedom in the mobile cloud.

The Free Software community has the opportunity to participate in the mobile cloud debate and shape this new environment. Ignoring the issues posed by the mobile cloud risks excluding a large number of digital citizens from the benefits that free software has brought to other computer users. The mobile cloud is an open territory where many vendors ${ }^{\underline{3}}$ are already fighting to lock-in users.

Resting on the cloud and network services, free and open source software should rely on licenses that prevent abuse. Fortunately, the Free Software Foundation has contributed a very good tool to bring freedom to the cloud. By extending its reach to interaction over a network, the Affero GPL $v 3^{4}$ (AGPLv3) is very effective at bringing copyleft to the services offered by cloud computing. Some people have had the chance to use open source software to offer services to the public, without returning anything to the community. That's taking open source software as free beer. It is just not being honest with the community, to the people who sweat to write the code to see someone running away with it and not contributing anything. Using the AGPLv3 for all software that can be used over a network is a smart way to start building a mobile cloud that respects user's freedom.

\begin{abstract}
About the author
Fabrizio Capobianco is an Italian entrepreneur now based in the Silicon Valley where he has founded Funambol Inc, the company behind the largest open source project in wireless, with over a million downloads, providing over the air data synchronization to a large number of mobile devices.
\end{abstract}

3 http://blog.internetnews.com/mmegna/2009/06/how-do-mobile-cloud-sync-servi.html

4 http://www.fsf.org/licensing/licenses/agpl-3.0.html 


\section{Licence and Attribution}

This paper was published in the International Free and Open Source Software Law Review, Volume 1, Issue 2 (December 2009). It originally appeared online at http://www.ifosslr.org.

This article should be cited as follows:

Capobianco, Fabrizio (2009) 'Five Reasons To Care About Mobile Cloud Computing', IFOSS L. Rev., 1(2), pp 139 - 142

DOI: 10.5033 /ifosslr.v1i2.24

\section{Copyright (C) 2009 Fabrizio Capobianco.}

This article was first published in the International In-house Counsel Journal.

This article is licensed under a Creative Commons UK (England and Wales) 2.0 licence, no derivative works, attribution, CC-BY-ND.

As a special exception, the author expressly permits faithful translations of the entire document into any language, provided that the resulting translation (which may include an attribution to the translator) is shared alike. This paragraph is part of the paper, and must be included when copying or translating the paper.

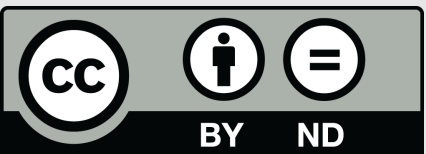

